immer nur an dem jeweiligen Berührungspunkt des Esters mit der verdünnten Säure vollzieht, zweitens aber auch die Möglichkeit in Erwägung zu ziehen ist, dass die Spaltung des Esters in mehreren Phasen unter Bildung von Monolein und Dioleïn verlaufen kann.

Eine einfache mathematische Formulirung dieses Processes, welcher als monomoleculare Reaction, wenn es gelänge, Oel und verdünnte Säure zu einer homogenen Lösung zu vereinigen, (wobei das Lösungsmittel sich natürlich nicht an der Reaction betheiligen dürfte) der oben angeführten Differentialgleichung entsprechen würde, ist deshalb nicht angängig.

Ueber die verseifende Wirkung von verdünnten Säuren auf andere Fettsäureester des Glycerins liegen Beobachtungen vor. So ist bekannt, dass der Glycerinester der Ricinusölsäure von Salzsäure leicht und nahezu vollständig gespalten wird. Wenn nun auch durch diese Versuche nachgewiesen ist, dass Salzsäure aus Trioleïn nur sehr geringe Mengen Glycerin abzuspalten vermag, so ist damit doch nicht ausgeschlossen, dass das beim Kochen der sulfurirten Oele entstehende Glycerin dem vorhandenen Trioleïn entstammt. Aus Gemischen von Triolein und Stearinschwefelsäure, wie solche nach meinen Versuchen in dem Einwirkungsproduct conc. Schwefelsäure auf Olivenöl vorliegen, kann beim Kochen mit Salzsäure die sich bildende Schwefelsäure verseifend auf das Triolein einwirken. Das Anwachsen der Säurezahl nach dem Kochen am Rückflusskühler kann ungezwungen nur durch die Bildung freier Oelsäure aus Trioleïn erklärt werden.
Wir haben:

\begin{tabular}{c||c|c|c}
\hline $\begin{array}{c}1 \text { g Oel } \\
\text { verbrauch. } \\
\text { K 0 H }\end{array}$ & $\begin{array}{c}\text { Ursprüngliche } \\
\text { Säurezahl }\end{array}$ & $\begin{array}{c}\text { Säurezahl nach } \\
\text { dem Kochen } \\
\text { mit HCl }\end{array}$ & Zunahme \\
\hline No. 1 & $0,0450 \mathbf{g}$ & $0,0740 \mathrm{~g}$ & $0,029 \mathbf{g}$ \\
No. 2 & $0,0117 \mathbf{g}$ & $0,0370 \mathbf{g}$ & $0,025 \mathrm{~g}$ \\
No. 3 & $0,0790 \mathbf{g}$ & $0,1030 \mathbf{g}$ & $0,024 \mathbf{g}$ \\
No. 4 & $0,0990 \mathbf{g}$ & $\mathbf{0 , 1 2 8 0} \mathbf{g}$ & $\mathbf{0 , 0 2 9} \mathbf{g}$ \\
No. 5 & $0,0470 \mathbf{g}$ & $\mathbf{0 , 0 7 0 0 ~} \mathbf{g}$ & $\mathbf{0 , 0 2 3} \mathbf{g}$ \\
No. 6 & $0,0836 \mathbf{g}$ & $\mathbf{0 , 1 0 8 0} \mathbf{g}$ & $0,024 \mathbf{g}$
\end{tabular}

Stearinschwefelsäure verbraucht zur Neutralisation 2 Molecüle $\mathrm{KOH}$. Beim Kochen mit Salzsäure geht aber diese in Oxystearinsäure über, welche nur noch ein Molecül $\mathrm{KOH}$ zur Neutralisation verbraucht. Würde also kein Glycerin abgespalten, so müsste die Säurezahl nach dem Kochen mit Salzsäure abnehmen, entsprechend den Mengen der vorhandenen Stearinschwefelsäure. Die bei diesen 6 Versuchen bemerkbar übereinstimmende Zunahme der Säurezahl von 0,025 lässt unter Berücksichtigung der gleich langen Kochdauer schliessen, dass durch die abgespaltene Schwefelsäure auch gleich viel Mengen Triolein verseift worden sind, so dass die übereinstimmende Zunahme der Säurezah! dadurch erklärt werden könnte. Bemerkenwerth dabei ist, dass der $\mathrm{SO}_{3}$-Gehalt der Oele No. 1 bis No. 4 stark differirt: No. $1=$ $4,8 \% \mathrm{SO}_{3}, \quad$ No. $2=1,08 \% \mathrm{SO}_{3}, \quad$ No. $3=$ $10,12 \% \mathrm{SO}_{3}$, No. $4=10,69 \% \mathrm{SO}_{3}$.

(Schluss folgt.)

\title{
Ueber die Verwendbarkeit des Aluminiums in der Stearinindustrie.
}

Von P. Pastrovich.

Obwohl bereits D on a th ${ }^{1}$ ) bei der Ueberprüfung der verschiedenen Angaben über das Verhalten des Aluminiums gegen Fette und Fettsäuren zu dem Ergebnisse gekommen ist, dass diese selbst in der Wärme und bei Luftzutritt nahezu ohne jede Einwirkung auf Aluminium sind, dieses Metall also als das in dieser Richtung widerstandsfähigste aller unserer technisch verwendeten Metalle angesprochen werden kann, dürfte es doch von Interesse sein, diesen Laboratoriumsversuchen auch Ergebnisse aus der Praxis gegenüberzustellen.

y) Dingler, polyt. Journ. 1895. 295. 18.
Bekanntlich werden die meisten der in der Stearinindustrie verwendeten Geräthschaften aus Kupfer oder Kupferlegirungen angefertigt, weil diese von allen anderen technisch verwendbaren Metallen noch am Besten der Einwirkung der Fettsäuren widerstehen. So werden unter Anderem auch die Platten der Stearinwarmpressen mit Mänteln aus circa $1 \mathrm{~mm}$ starkem Kupferblech versehen, um einerseits die eisernen Pressplatten vor dem Angriff der heissen 'Fettsäuren und diese selbst vor der Verunreinigung durch Eisenseifen zu schützen, andrerseits die Etweindelles vor dem zu schnellen Verbrennen zu bewahren. Solche Kupferblechmäntel werden, 
abgesehen von ihrer verhältnissmässigen Kostspieligkeit, selbst bei sehr ökonomischer $\mathrm{Be}$ handlung schon nach 7 bis 8 Jahren unbrauchbar, da sie nach dieser Zeit durch die Einwirkung der Fettsäuren in der Wärme und bei Luftzu. tritt vollständig durchgefressen werden; ausser dem deformiren sich dieselben in Folge der wiederholten grossen Druckwirkungen, so dass die Ränder öfters beschnitten werden müssen.

Um vorerst ein Bild über die verschiedene Angreifbarkeit des Kupfers gegenüber dem Aluminium und dann darüber zu erhalten, ob es zweckmässig sein würde, ersteres durch letzteres zu ersetzen, wurden je eine Kupfer- und Aluminiumplatte von denselben Dimensionen $(1500 \times 520 \times 1 \mathrm{~mm})$ gewogen und in dieselbe hydraulische Warmpresse eingesetzt; die Presse wurde täglich durchschnittlich elf Mal beschickt. Nach genau zwei Jahren wurden die beiden Versuchsmäntel aus der Presse genommen, durch wiederholtes Abbürsten mit einer Strohbürste in heissem Wasser gereinigt und nach dem $\mathrm{Ab}$ reiben mit trockenen Tüchern die Gewichts. verluste bestimmt. Das Kupferblech, Anfangsgewicht $7,62 \mathrm{~kg}$, wog nachher $6,42 \mathrm{~kg}$, das Aluminiumblech, Anfangsgewicht $2,26 \mathrm{~kg}$, wog nachher $2,22 \mathrm{~kg}$; das Kupfer hatte also $1,20 \mathrm{~kg}$ oder $15,75 \%$, das Aluminium nur $0,04 \mathrm{~kg}$ oder $1,77 \%$ an Gewicht verloren. Die Dehnung des Kupferbleches betrug in der Länge $10 \mathrm{~mm}$, in der Breite $5 \mathrm{~mm}$, während am Aluminiumblech keine Aenderung der Dimensionen constatirt werden konnte.

Auf Grund dieses überaus günstigen Ergebnisses wurde für eine ganze Warmpresse eine Garnitur von 28 Stück Aluminiumschutzblechen angeschafft. Dieselbe wog $67,40 \mathrm{~kg}$ und erlitt nach abermals zweijähriger Inanspruchnahme einen Gewichtsverlust von $0,920 \mathrm{~kg}$ oder $1,37 \%$. Dabei zeigten sich die Aluminiumbleche nicht im Mindesten angefressen und hatten keine Deformation erlitten, trotzdem das Metall so weich gewählt worden war, dass sich die Bleche in der Kälte im rechten Winkel biegen liessen, ohne zu reissen. - Eine Garnitur aus neuen Kupferblechen im Gewichte von $227,85 \mathrm{~kg}$, welche im April 1894 in eine Warmpresse montirt worden war, hatte im Juni d. J., wo sie sich als total unbrauchbar erwies, das Gewicht von $10.3 \mathrm{~kg}$; die Abnutzung betrug also $124,50 \mathrm{~kg}$ oder $54,73 \%$. Unter Zugrundelegung einer nur $50 \%$ igen Abnutzung bis zur Unbrauchbarkeit würde sich also die Verwendungsdauer der Aluminiumblechmäntel aus den oben gewonnenen Daten auf ca. 66 Jahre berechnen.

Ein weiteres in Betracht zu ziehendes Moment ist der Unterschied in den Gestehungskosten, welche sich bei den heutigen Preisen, K. 500,für $100 \mathrm{~kg}$ Aluminiumblech und K. 194.- für Kupferblech, für einen Aluminiummantel von $1500 \times 250 \times 1 \mathrm{~mm}$ im Gewichte von $2,26 \mathrm{~kg}$ auf K. 11,30, für einen gleich dimensionirten Kupfermantel von $7,50 \mathrm{~kg}$ auf $\mathrm{K} .14 .74$ be laufen, so dass also eine Aluminiumgarnitur um rund $23 \%$ billiger zu stehen kommt, als eine Kupferblechgarnitur.

Sonstige Geräthe, wie Schöpfer, Kruken etc., überhaupt alle diejenigen, welche ohne Herstellung einer Löthnaht verfertigt werden können, erweisen sich den gleichen Geräthschaften aus Kupfer gegenüber, gegen Fettsäuren im Gebrauche weit haltbarer.

Es wäre noch von Interesse zu erfahren, wie sich das Aluminium gegen die länger dauernde Einwirkung von Fettsäuredämpfen, also in Destillirblasen verhält; als Futter für Autoclaven dürfte es wohl wegen der Gegenwart der wenn auch geringen Mengen von Alkalien in den zur Fettspaltung benutzten alkalischen Erden (Kalk und Magnesia) und seines Verhaltens gegen siedendes Wasser und hoch. gespannten Wasserdampf nicht zu verwenden sein. Für die gewöhnlichen Operationen jedoch ist das Aluminium das Metall par excellence der Stearinindustrie.

\title{
Verwerthung der Walkwässer.
}

\author{
Von F. Kasoler.
}

In den ästerreichischen Textilstädten, insbesondere im österreichischen ,Manchester.s, Brünn, hat sich ip Verbindung mit der Tuch fabrikation eine Industrie ausgebildet, wie wir sie in demselben Umfange wohl in keinem andern Lande des Continents finden. Es ist die Verwerthung und Aufarbeitung der beim Walken der Tuche resultirenden Seifenwässer.

Wie bekannt, werden die Wollen zum Zwecke des Spinnens gefettet, um sie geschmeidiger zu machen. Diese Procedur wird mit dem Ausdruck *Spicken * oder 